\title{
New Fourier CGH coding using DMD generated masks
}

\author{
Frederic Zamkotsian ${ }^{1}$, Giorgio Pariani ${ }^{2}$, Patrick Lanzoni ${ }^{1}$, \\ Luca Oggioni ${ }^{2}$, Chiara Bertarelli ${ }^{3}$, Andrea Bianco ${ }^{2}$ \\ ${ }^{1}$ Aix Marseille Université, CNRS, CNES, LAM (Laboratoire d'Astrophysique de Marseille), 13388, Marseille, France \\ ${ }^{2}$ INAF - Osservatorio Astronomico di Brera, Via Bianchi 46, 23807 Merate, Italy \\ ${ }^{3}$ Politecnico di Milano, Dipartimento di Chimica, Materiali e Ingegneria, p.zza L. Da Vinci 32, 20133, Milano, Italy \\ e-mail: frederic.zamkotsian@lam.fr
}

\begin{abstract}
Computer Generated Holograms (CGHs) are powerful optical elements used in many fields, such as wavefront shaping, quality testing of complex optics and anti-counterfeiting devices. Lee algorithm is the most used to generate binary amplitude Fourier holograms. Grayscale CGHs are known to give a higher reconstruction quality than binary holograms, but they usually require a cumbersome production process. A very simple and straightforward method of manufacturing rewritable grayscale CGHs is here proposed by taking advantage of two key components: a Digital Micro-mirror Device (DMDs) and a photochromic plate. An innovative algorithm, named Island algorithm, able to generate grayscale amplitude Fourier CGHs, is reported and compared with the standard Lee approach, based on 9 levels. A crucial advantage lies on the fact that the increase or decrease of the quantification does not affect the spatial resolution. In other words, the new coding leads to a higher spatial resolution (for a given CGH size) and a reconstructed image with an order of magnitude higher contrast with respect to the classical Lee-coded hologram. In order to show the large potential of our approach, a 201 levels Island hologram is designed, produced and reconstructed, pushing the contrast to values higher than $10^{\wedge} 4$. These results reveal the high potential of our process as well as our algorithm for generating programmable grayscale CGHs. Grayscale objects are also studied in order to be produced with our new coding scheme: simulations show a much better reconstruction (resolution, fidelity, contrast) thanks to the quantification of the transparency than the Lee algorithm commonly used.
\end{abstract}

Key words: Computer Generated Hologram, CGH, Fourier coding, programmable CGH, photochromic material, optical testing, wavefront shaping, DMD.

\section{INTRODUCTION}

Wavefront shaping, complex optics testing, including aspherical and free-form optics or optical instrument alignment techniques will greatly benefit from Computer Generated Holograms (CGHs) [1]. They are classified in two groups:

1- phase holograms, which are obtained by recording a phase variation in a material having a modulated refractive index or thickness;

2- amplitude holograms, where an intensity pattern is recorded in a material whose transparency can be locally controlled. Phase and amplitude holograms provide the same performances in terms of image reconstruction quality, but different diffraction efficiency. For instance, binary phase holograms, show $40 \%$ diffraction efficiency in the first order, whereas efficiency is limited to $10 \%$ for binary amplitude holograms [2]. Therefore, amplitude holograms are usually applied in interferometry, which is not intensity limited.

Grayscale amplitude and grayscale phase holograms are known to give a higher reconstruction quality than binary holograms [2], but they require a more complex production process. Specifically, the production of phase grayscale CGHs is complex since a series of masks has to be consecutively aligned very precisely, and a developing step is required after each exposure step to obtain the final hologram.

To our best knowledge, only grayscale phase CGHs have been obtained so far by micro-lithography [3], the uniformity of the material thickness being the main limiting parameter for these components [4]. Concerning amplitude CGHs, they are nowadays produced in chrome on glass by means of lithographic techniques, either mask or maskless (by direct 
writing) lithography. Due to the binary nature of the chrome developing process, these techniques allow for easily writing binary CGHs, but they cannot provide grayscale CGHs.

We previously demonstrated an original recording technique, which makes use of a programmable mask and a nonthreshold photosensitive material, to produce ready to use grayscale CGHs in a one exposure process without requiring any developing step [5,6]. Indeed, a set-up based on a Digital Micro-mirror Device (DMD), which has been originally developed to generate programmable slit masks in multi-object spectrographs [7], is considered. DMDs are programmable devices, composed of millions of micro-mirrors reconfigurable in real time. Actually, DMDs have been extensively used to generate dynamic binary or grayscale holograms [8], by exploiting the fast switching of the mirrors at frequencies higher than the human vision frame rate. The grayscale originates as a dynamic effect and not as a steady state effect. However, the discrete structure of the device induces a high scattering and background noise from the mirrors edges when illuminated with laser light [9], making such holograms useless for interferometry and metrology. Nevertheless, DMDs perfectly reproduce binary masks to be projected with incoherent light on photosensitive plates, thus producing amplitude CGHs. In this work, such plate consists in a photochromic film that can be reversibly converted from an opaque and colored form to a transparent form upon exposure with light of suitable wavelengths [10].

Actually, reversible holograms have been already obtained with photochromic materials [11, 12] and real-time photochromic holograms were shown, by exploiting the fast transition of imidazole dimers [13, 14]. Moreover, photochromic binary CGHs for optical testing have been recently demonstrated [15]. In photochromic materials, a ready to use hologram is generated just after the light exposure, and the reversibility of the photoconversion makes devices rewritable. Even more interesting, the transparency of a photochromic layer can be tuned by the dose of light absorbed, which opens to the development of grayscale patterns [16]. In fact, the DMD set-up allows for easily recording grayscale CGHs in a single exposure process. The lower diffraction efficiency of grayscale amplitude holograms with respect to binary amplitude holograms (6\% vs. $10 \%[2])$ is here compensated by a better image reconstruction quality, an easy exposure process and no developing steps, which are the limiting factors in the production of grayscale phase holograms.

Previously, we have successfully recorded the very first amplitude grayscale CGH, in equally spaced levels, so called stepped CGH. We recorded up to 1000x1000 pixels CGHs with a contrast greater than 50, using Fresnel coding scheme. Fresnel's CGH are obtained by calculating the inverse Fresnel transform of the original image at a given focus, ranging from $50 \mathrm{~cm}$ to $2 \mathrm{~m}$. The reconstruction of the recorded images with a $632.8 \mathrm{~nm} \mathrm{He}-\mathrm{Ne}$ laser beam leads to images with a high fidelity in shape, intensity, size and location. These results reveal the high potential of this method for generating programmable/rewritable grayscale CGHs, which combine DMDs and photochromic substrates. $[5,17]$

In this paper, we report innovative results for Fourier holograms: a new family of Fourier holograms named Island CGHs, including an original coding algorithm, leading to higher spatial resolution and a reconstructed image with a much higher resolution, a better compacity and an increased throughput, in comparison with the classical Lee-coded holograms. Grayscale objects are also studied in order to be produced with our new coding scheme.

\section{RECORDING AND RECONSTRUCTION OF A CGH}

Two set-ups have been developed for the recording of the calculated CGH and for the reconstruction of the original encoded images.

\subsection{Recording set-up}

Figure 1 shows our recording set-up, dedicated to the CGH recording on the photosensitive plate. The DMD, controlled by the formatter board [18] is illuminated by a collimated beam from a white source, and redirects the light toward the plate. The beam is illuminating the entire DMD and the light power is homogeneous on the plate. The pattern reproduced by the DMD has to be projected onto the plate as precisely as possible, so the plate is illuminated through an Offner relay with a magnification of 1:1. This relay provides a nearly aberration free beam and has the advantage of being compact. The unit magnification means that the maximum size of CGH is directly limited by the size of the DMD; the micromirrors of the DMD therefore correspond to the "pixels" of the CGH. Finally, a post-CGH imaging system located right after the CGH plate consists of two lenses, a filter around $600 \mathrm{~nm}$ and a CCD camera. This system in an afocal assembly allows imaging of the CGH during writing, in situ and in real time. Magnification is tuned by changing properly the pair of lenses, from a value of 1 up to 4 . 
DMD, CGH and camera planes are then conjugated. Note that DMD plane is a tilted focal plane due to the nature of micro-mirror array: each micro-mirrors tilts out of the array plane by $12^{\circ}$, leading to a global $24^{\circ}$ tilted focal plane. This effect is reproduced as well at the post-CGH imaging camera level (the camera plane is tilted by $24^{\circ}$ ).

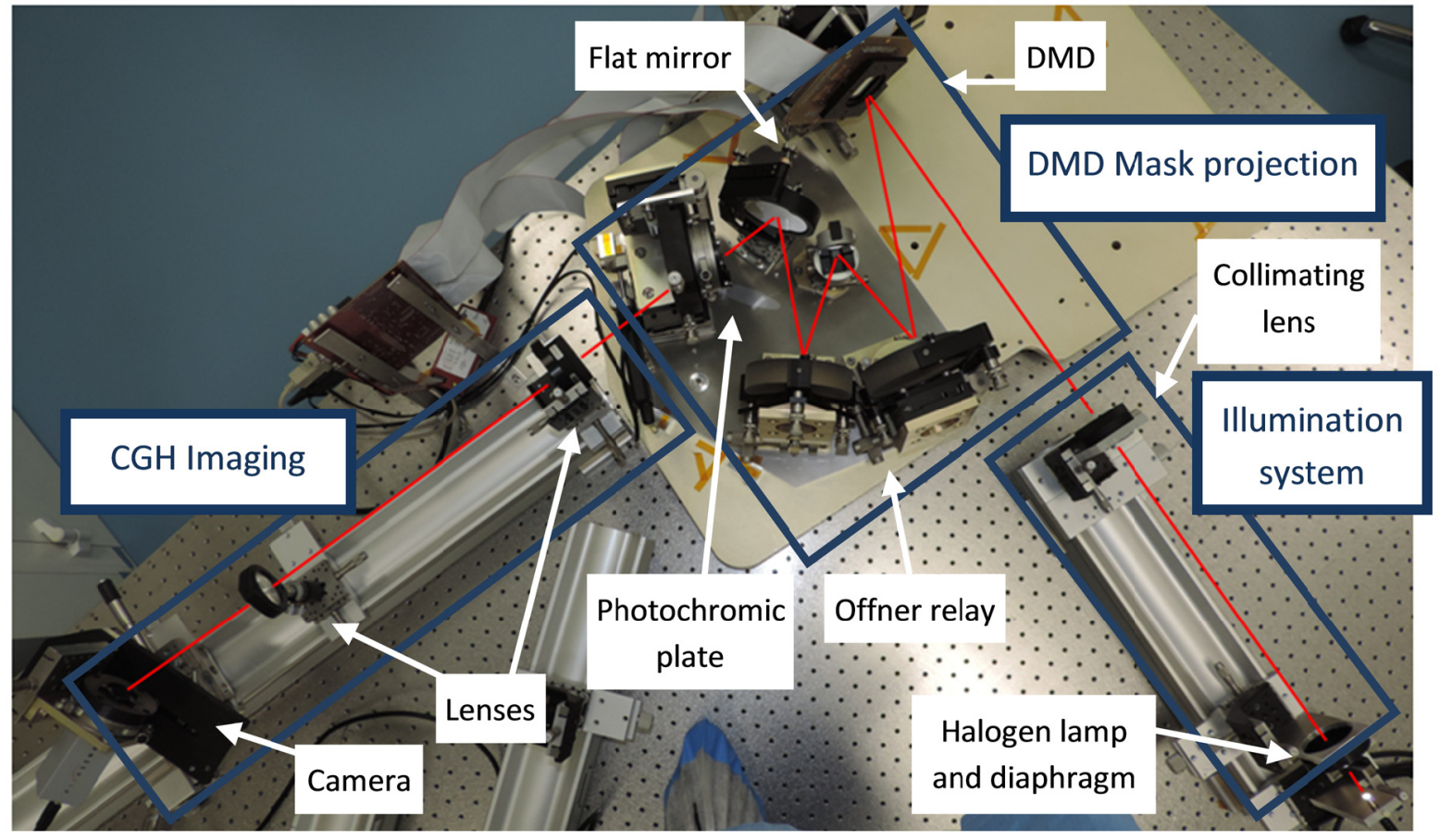

Fig. 1: Picture of the set-up dedicated to CGHs recording; it is based on an illumination unit towards the DMD, an imaging optical system based on a 1:1 magnification Offner relay from the DMD plane to the CGH plane, and a post-CGH imaging system.

\subsubsection{Mask generator}

Digital Micromirror Devices (DMD) from Texas Instruments could act as reconfigurable mask generator. The largest DMD chip developed by TI features 2048 x 1080 mirrors on a $13.68 \mu \mathrm{m}$ pitch, where each mirror can be independently switched between an $\mathrm{ON}\left(+12^{\circ}\right)$ position and an OFF $\left(-12^{\circ}\right)$ position (Fig. 2). This component has been extensively studied in the framework of an ESA technical assessment of using this DMD component (2048 x 1080 mirrors) for space applications (for example in EUCLID mission). Specialized driving electronics and a cold temperature test set-up have been developed. Our tests reveal that the DMD remains fully operational at $-40^{\circ} \mathrm{C}$ and in vacuum. A 1038 hours life test in space survey conditions $\left(-40^{\circ} \mathrm{C}\right.$ and vacuum) has been successfully completed. Total Ionizing Dose (TID) radiation tests, thermal cycling (over 500 cycles between room temperature and cold temperature, on a non-operating device) and vibration and shock tests have also been done; no degradation is observed from the optical measurements. These results do not reveal any concerns regarding the ability of the DMD to meet environmental space requirements [18].

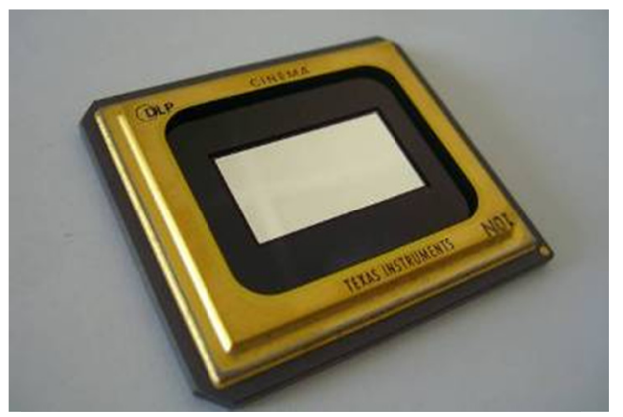

Fig. 2: DMD chip from Texas Instruments (2048 x 1080 micromirrors). 


\subsubsection{DMD mask projection}

A simple imaging Offner-type layout has been set up, based on two identical spherical concave mirrors and a convex mirror (Fig. 3). Indeed, spherical mirrors belong to the same mother mirror, sharing the same radius and center of curvature. One mirror only could be used instead of two separate ones, helping the alignment of the system. However, mechanical and operational constraints can lead to split these two mirrors. We have preferred two identical spherical mirrors with a diameter of $160 \mathrm{~mm}$ and a radius of curvature of $438 \mathrm{~mm}$. The convex mirror has a $224 \mathrm{~mm}$ radius of curvature. [19]

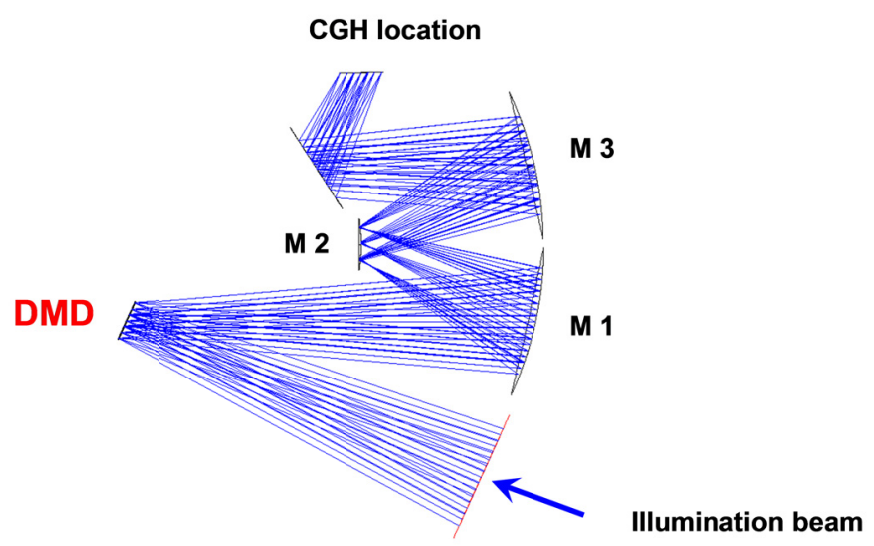

Fig. 3: Optical layout of the imaging set-up.

This optical layout will make the system simple and efficient. Additionally it will not suffer from chromatic aberrations. Delivered image quality onto the CGH plane is high enough to not degrade spatial resolution. Typical monochromatic spot diameters are $<20 \mu \mathrm{m}$ over the whole FOV for wavelengths between $400 \mathrm{~nm}$ and $800 \mathrm{~nm}$.

\subsection{Reconstruction set-up}

The reconstruction set-up, shown in Fig. 4, is dedicated to CGH reconstruction. The source is a collimated $632.8 \mathrm{~nm}$ HeNe laser. The camera, placed at the distance $\mathrm{z}$ from the plate, is illuminated through the $\mathrm{CGH}$. As described in the next section, the Fresnel holograms are directly calculated from the propagation equations of light, and they are for a given focus. It is thus not necessary to add a lens between the plate and the camera to reconstruct the image, unlike with the Fourier holograms. This is why the source should be rigorously collimated, because a variation in the quality of the collimation can displace the focal plane along the optical axis and change the size of the encoded image.

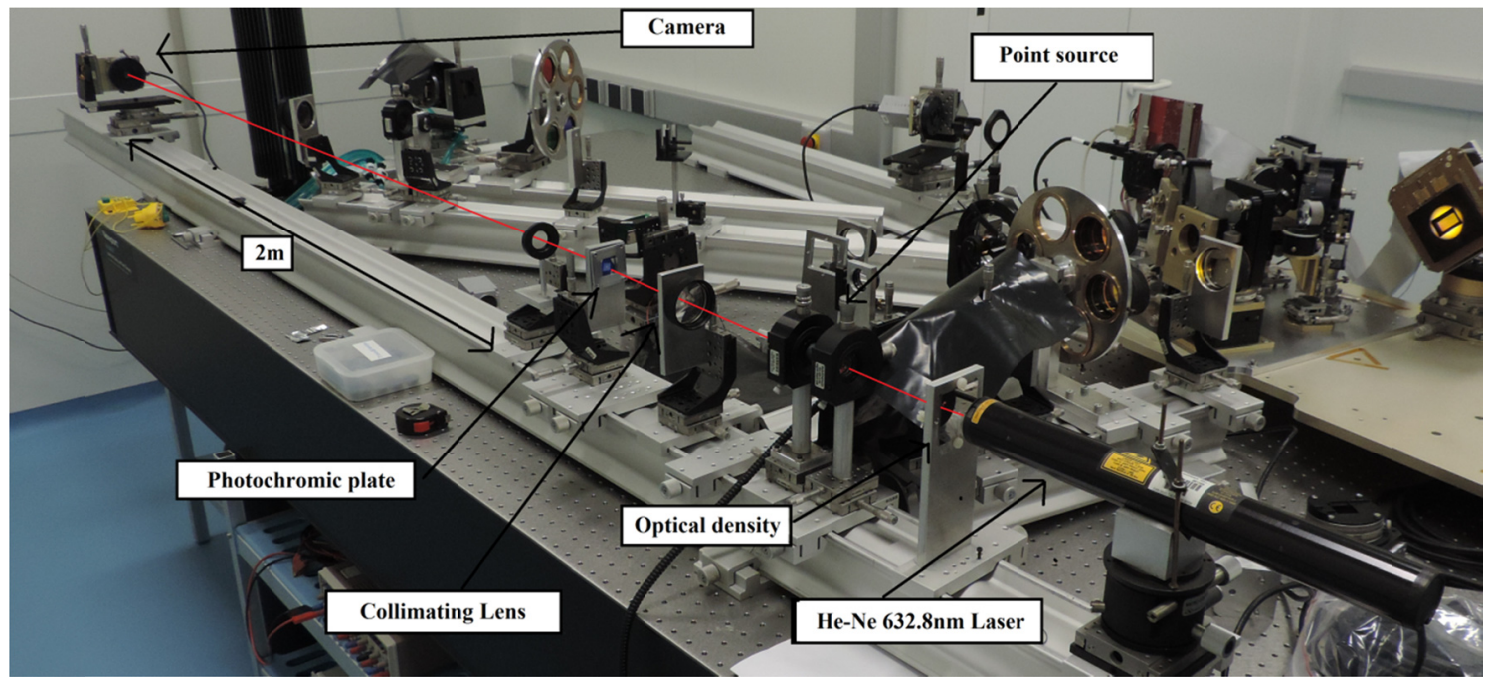

Fig. 4: Picture of the set-up dedicated to image reconstruction, including a $632.8 \mathrm{~nm} \mathrm{He}-\mathrm{Ne}$ laser, the CGH plane and the camera located at the hologram projection distance ( $2 \mathrm{~m}$ in this case) for Fresnel CGHs.

For Fourier holograms, an additional collimating lens is required. 


\section{PHOTOCHROMIC PLATES}

The photosensitive material is a diarylethene-based polyurethane [20]. Such kind of photochromic substrate contains a large content of photochromic units (i.e. $50 \%$ wt. in this work) that turns into a high contrast between the colored and uncolored forms, also for thin films. This layer can be reversibly converted from an opaque and colored form to a transparent form upon exposure with light of suitable wavelengths, around 600nm (Fig. 5). Moreover, the optical quality in terms of transparency and scattering is very good. The photochromic plate is $3 \mu \mathrm{m}$ thick and the film is set uniformly to the opaque state by a UV exposure. The conversion under light illumination of the photochromic film has been previously determined by a kinetic model that provides the degree of transparency as function of the film features and illumination conditions [16].

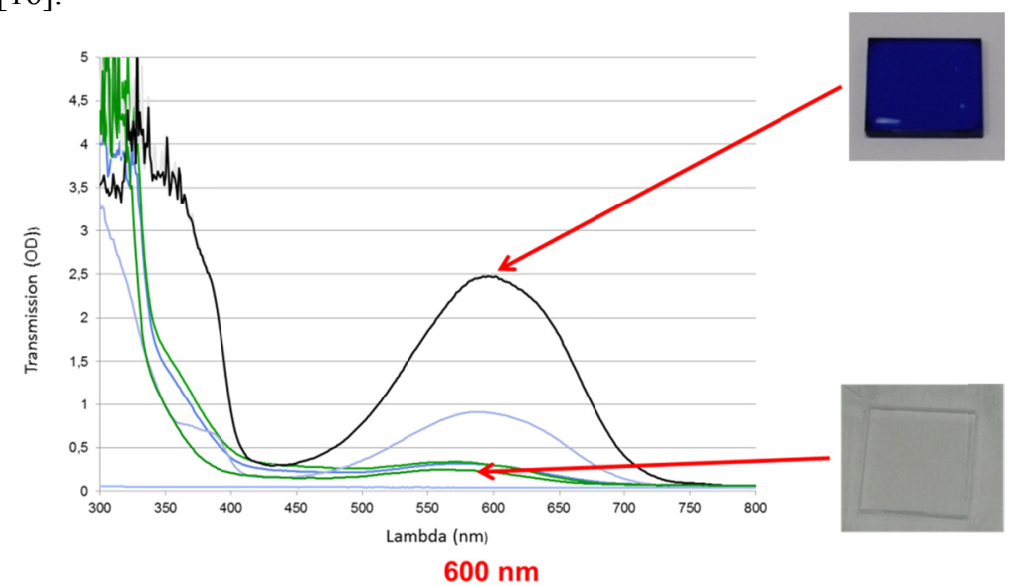

Figure 5: Transmission (Optical Density) of the photochromic material used in the UV-Visible range of the spectrum showing the opaque to the transparent states, according to the illumination amount of the plate

\section{FOURIER CGH}

Fourier holograms are obtained using algorithms encoding the inverse Fourier transform of the wavefront to be reproduced. These algorithms [21, 22, 23] are operating in a common way. Once the inverse Fourier transform is calculated, the complex information contained in each pixel is encoded in a cell of $\mathrm{WxH}$ pixels, $\mathrm{W}$ and $\mathrm{H}$ depending on the used algorithm. Lohmann, Lee and Burkhardt algorithms, all generate binary CGH with compactness and quantification of information at different levels. The Lee algorithm is the most widely used and will be considered as a reference for the current work. Only binary Fourier holograms have been produced using these algorithms. The number of pixels of a Fourier hologram is therefore necessarily larger than the number of pixels defining the image. This leads to limiting the resolution and size of images to be encoded. Since these holograms are based on the Fourier transform, it is necessary to add a lens after the $\mathrm{CGH}$ in the reconstruction set-up, for applying the optical Fourier transform to the CGH, and display the image.

\subsection{The Lee algorithm}

The algorithm proposed by Lee is encoding the real and imaginary parts of the complex Fourier function (Fig. 6a) instead of the phase and amplitude [24]. The binary version of the algorithm, which is the only one developed up to now, foresees a $4 \times 4$ elements cell for each pixel and encodes the real and the imaginary part of the complex number in two times two columns (separating the positive and the negative values), leading to 9 quantification levels ( 8 discrete levels plus the zero position), as shown in the Fig. 6 b.
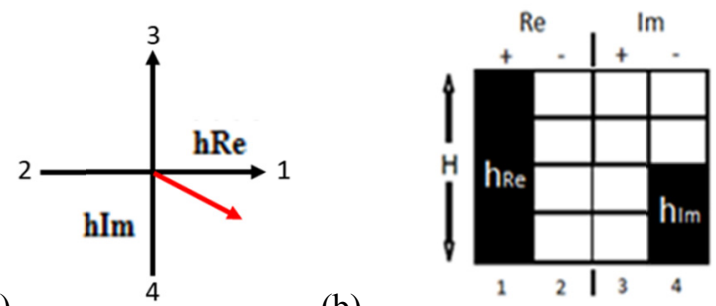

(b) . .

(c)

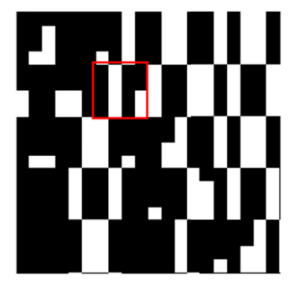

Fig. 6: (a) Complex number to be encoded; (b) correspondent cell of the Lee algorithm; (c) example of a Lee hologram 
The only way to increase the number of quantification levels is to increase the size of the cell, $8 \mathrm{x} 8$ elements cell for 33 levels, and 16x16 elements cell for 129 levels, increasing then the hologram size. An example of a Lee hologram is provided in Fig. 6c.

\subsection{The Lee-compact algorithm}

The grayscale approach proposed in this paper opens to new possibilities to increase the hologram information density and hence the reconstruction fidelity against binary holograms of the same size and resolution.

Our first newly proposed algorithm uses the grayscale approach to pop-up the columns of Lee cells, squeezing the information of a quadrant of the complex plane into a single element (Lee-Compact). As shown in Fig. 7b, the cell is composed by $2 \times 2$ elements, representing the positive and negative components of the real and imaginary parts of the complex function. Lee-compact algorithm gives better results than the binary Lee algorithm in terms of compactness and quantification levels, but shows small diffraction efficiency since two pixels are intrinsically black.

The number of discrete levels available with this algorithm is infinite, limited by the recording medium to display these levels. This is done without changing the size of the elementary cell.

An example of a Lee-compact hologram is provided in Fig. 7c.

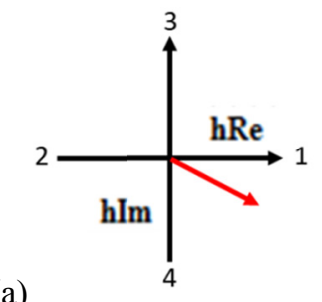

(b)

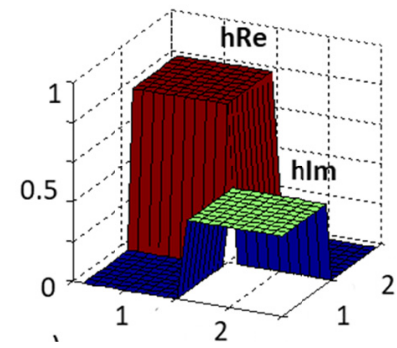

(c)

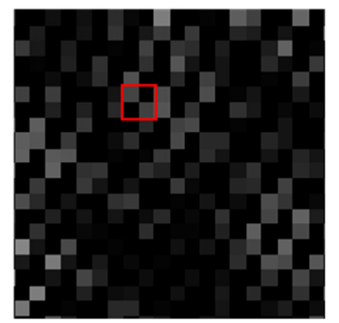

Fig. 7: (a) Complex number to be encoded; (b) correspondent cell of the Lee-compact algorithm;

(c) example of a Lee-compact hologram

\subsection{The Island algorithm}

In order to overcome the limitation due to the two intrinsically black pixels of the Lee-compact algorithm, we introduce a new coding scheme (named Island), where the cell is composed by $2 \times 1$ elements correspionding to the real and the imaginary parts of the complex number. An offset is added to obtain positive values only that can be transformed into transparency levels (Fig. 8b). However, we still keep coding the information in a $2 \times 2$ elements cell to avoid anamorphism in the reconstructed image.

The number of discrete levels available with this algorithm is also infinite, limited by the recording medium to display these levels. This is done without changing the size of the elementary cell. For example, let's consider a CGH with 129 quantification levels; a 256-elements pixel is required with the Lee-code, while the size of the pixel always relies on a $2 \times 2$ element cell with the Island code; this is therefore 64 times more compact than the Lee-code in the case of 129 quantification levels.

An example of an Island hologram is provided in Fig. 8c.
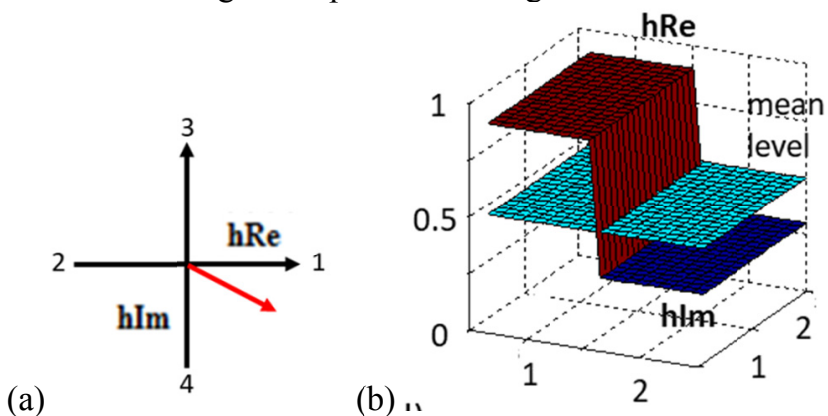

(c)

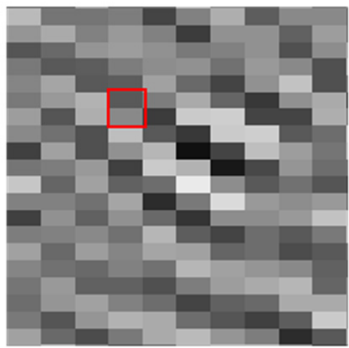

Fig. 8: (a) Complex number to be encoded; (b) correspondent cell of the Island algorithm; (c) example of an Island hologram 


\section{ISLAND CGH FOR BINARY IMAGES}

\subsection{Island CGH for binary images calculation}

We chose as object the logo of the astronomical instrument BATMAN project as shown in Fig. 9a [19], coded on 256 x 256 pixels, for a CGH of 1000x 1000 pixels, with an actual size of $14 \mathrm{~mm}$. The actual size of the CGH is limited by the size of the DMD device used for the CGH recording, see paragraph 2. The size of the object to be reconstructed must be smaller than the size of the image to be encoded. For a better balance between the frequemcy and the spatial domain, we set a ratio of one third between the object size and the image size; the logo is not centered in order to locate the reconstructed image as far as possible from the $0^{\text {th }}$ order signal, increasing the SNR.
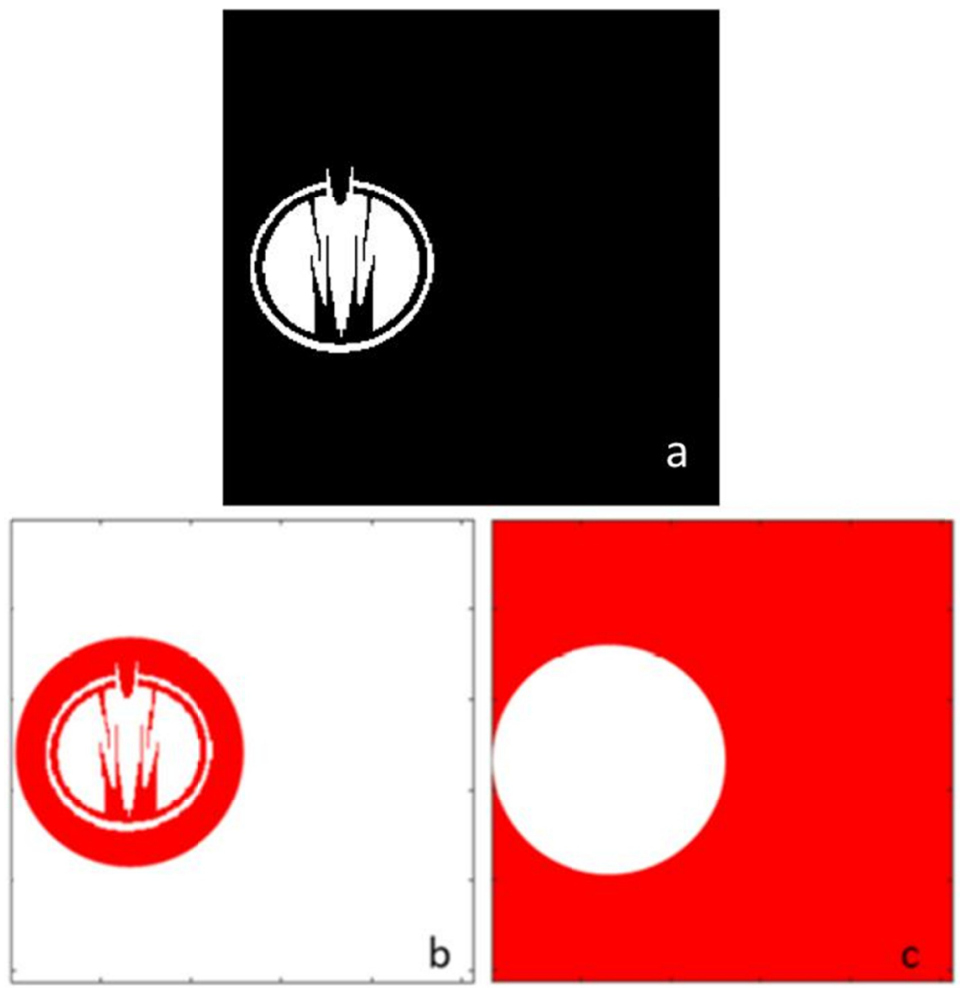

Fig. 9: (a) BATMAN logo original image; regions where parameters are calculated, in red: neighborhood (b), and background (c).

Simulations were performed to evaluate the performances of the two different coding strategies. We considered:

1. the Lee coding, with 4 by 4 elements cell, corresponding to 9 quantification levels for the real part of the complex Fourier function and 9 for the imaginary part,

2. the Island coding with the same number of gray tones.

In both cases, we kept constant the size of the hologram at 512 by 512 pixels. To be consistent, the size of the image to be reconstructed was set to 128 by 128 pixels for the Lee coding, and 256 by 256 pixels for the Island coding.

We applied a random phase between 0 and $2 \pi$ to the image before the hologram calculation to reconstruct all the spatial frequencies of the image. The complex hologram is calculated by the Inverse Fast Fourier Transformation (IFFT) of the complex image. The complete description of our calculation procedure is given in [6].

Original images for the Lee 4 by 4 (128 by 128 pixels) and the Island 2 by 2 (256 by 256 pixels) are shown in Fig. 10(a) and 10(b). The holograms encoded with the Lee and Island algorithms are reported in Fig. 10(c) and 10(d), respectively, together with the simulated reconstructed images in Fig. 10(e) and 10(f). Note the average vallue at the mid-gray level for the Island hologram. 

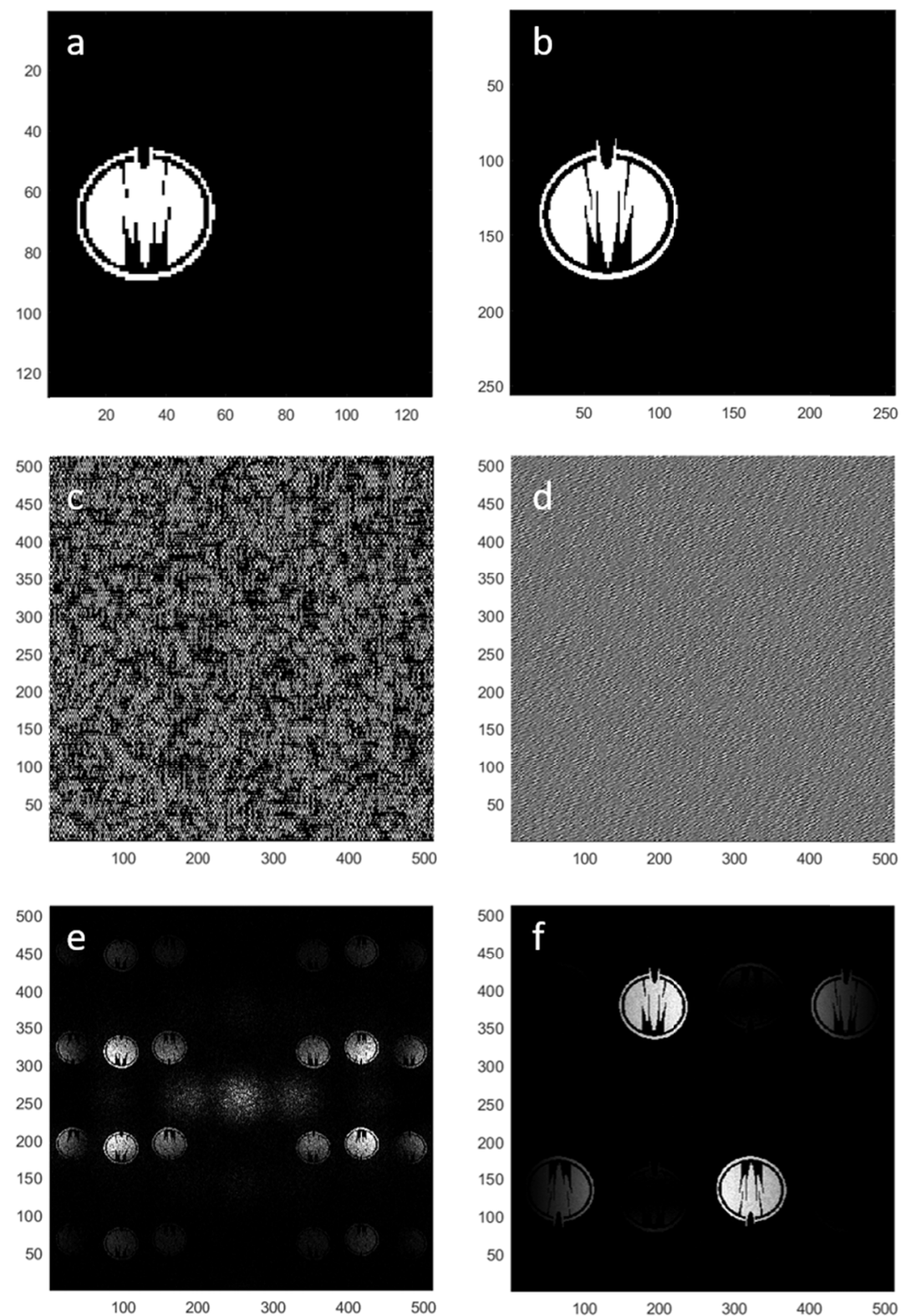

Fig. 10: Original images, holograms, and reconstructed images for the Lee 4 by 4 with $f=4$ (a, c, e) and the Island 2 by 2 with $\mathrm{f}=2(\mathrm{~b}, \mathrm{~d}, \mathrm{f})$, both coded with 9 quantification levels.

For a comparison of the reconstructed image quality, we considered the following parameters:

- the local contrast, defined as the signal to noise ratio $(\mathrm{S} / \mathrm{N})$ between the object and its neighborhood (Fig. 9(b)), calculated as the ratio between the average intensity of the object and the background in a circle with a diameter 1.5 times that of the object;

- the global contrast, defined as the signal to noise ratio (S/N) between the object and the background (Fig. 9(c)), calculated as the ratio between the average intensity of the object and of the background;

The Island coding behaves much better than the Lee coding under all aspects: while the correlation is similar (the image is reconstructed fairly well in any case), the $\mathrm{S} / \mathrm{N}$ is larger than two orders of magnitude in the Island coding, meaning that the image is better defined and the contrast between light and dark regions is greater. The summary of the results is reported in Table 1. With the increase of the quantification levels, the $\mathrm{S} / \mathrm{N}$ ratios dramatically rise. 
Table 1: performances of the two different codings for 9 levels and 201 levels.

\begin{tabular}{|l|c|c|}
\hline & S/N neighborhood & S/N background \\
\hline Lee, 9 levels & 23 & 24 \\
\hline Island, 9 levels & 314 & 720 \\
\hline Island, 201 levels & 30000 & 3600 \\
\hline
\end{tabular}

\subsection{Island CGH for binary images recording}

The BATMAN logo is recorded using the set-up and the procedure discussed in paragraph 2. Grayscale CGHs are generated by a set of masks applied to the DMD, and sequentially projected onto the recording plate. Because of the nonlinear response of the photochromic material, a calibration curve of the photosensitive plate is measured, and each mask is projected for a different time in order to get a linear grayscale. The addition of all masks permits to reach the required level of transparency for each pixel of the CGH. The BATMAN logo has been recorded with a set of 201 masks.

The plates used to record the CGH consisted in a photochromic thin film deposited on $3 \mathrm{~mm}$ thick glass substrates. The active component is a diarylethene molecule [20], namely the 1,2-bis(2-methyl-5-dimethylaminophenyl) perfluorocyclopentene dispersed in $\mathrm{CAB}$ (cellulose acetate butyrate) polymer matrix, with a concentration of $16.6 \% \mathrm{wt}$.

\subsection{Island CGH for binary images reconstruction}

The image was reconstructed with the reconstruction set-up (paragraph 2), by means of a collimated beam at $632.8 \mathrm{~nm}$ and it is shown in Fig. 11. The reconstructed image is including the $0^{\text {th }}$ order (continuum, horizontal and vertical spikes) and the $1^{\text {st }}$ order diffracted beams (reconstructed images). The dashed lines tilted by $45^{\circ}$ are diffraction patterns due to the DMD structure (tilting mirrors along their diagonals).

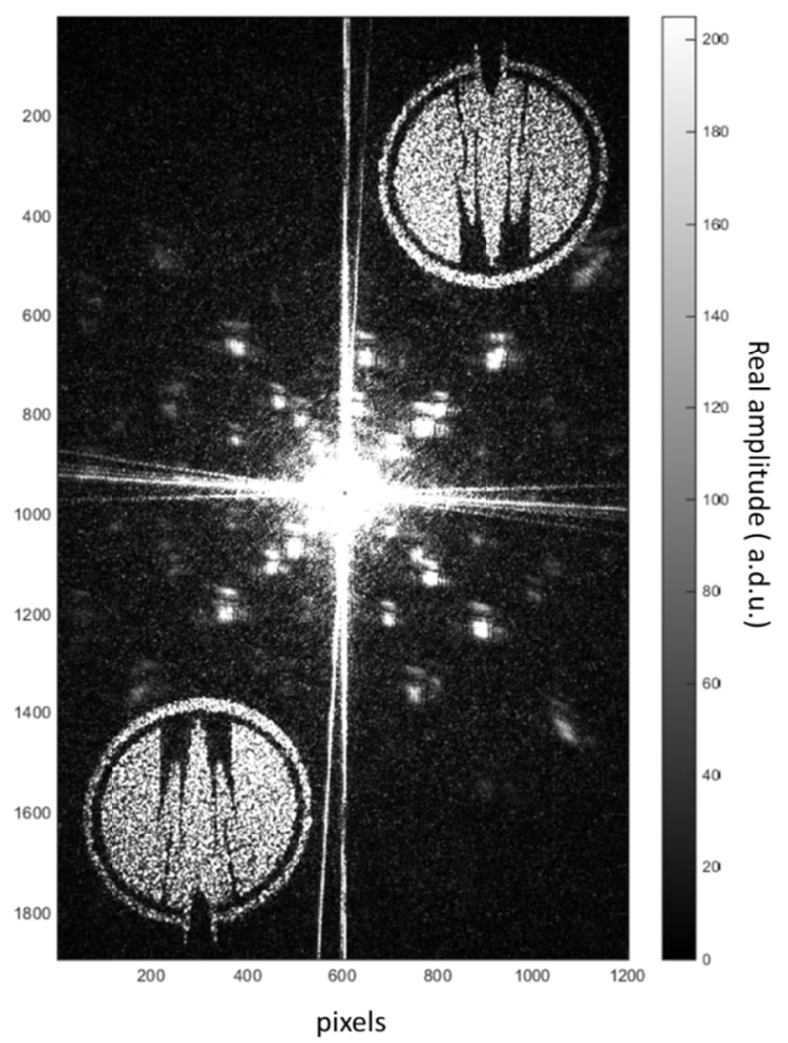

Fig. 11: Reconstructed image of the Island CGH (object: BATMAN project logo) 
The Fig. 12 is a comparison of the original image (Fig. 9a) and the reconstructed one (Fig. 11).

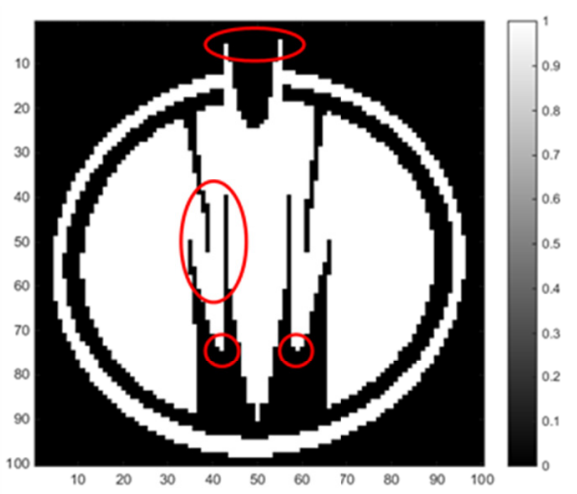

pixels

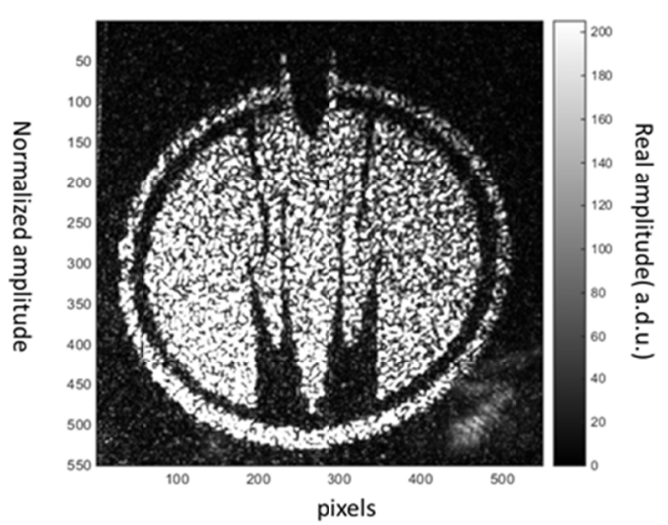

(b)

Fig. 12: (a) Original and (b) reconstructed images. Red marks show that every details of the image are perfectly reproduced. Single pixels and one pixel wide lines appear clearly on the reconstructed image.

Speckles are inevitable since the source is coherent but the brightness is almost homogeneous. The resolution of the image is enough to make the edge look round and not pixilated. Structures are remarkably faithful to originals, reproduced pixel by pixel as the red circles on the Fig. 12a bring out. The two little circles show that one pixel coming out of a bigger structure is visible on the reproduction. The ellipse that surrounds the top of the image shows that one pixel wide lines of white pixels are clearly reproduced, and the other ellipse shows the same result for one pixel wide lines of black pixels inside structures.

These results show that our Island algorithm generates grayscale holograms that give much more compact CGH then the Lee algorithm commonly used, and a better reconstruction (resolution, fidelity, contrast) thanks to the quantification of the transparency.

\section{ISLAND CGH FOR GRAYSCALE IMAGES}

Island coding for grayscale images may also exhibit a much better result with respect to the Lee algorithm. Comparison of simulated reconstructed images with Lee and Island coding is presented in this paragraph.

We chose as object a dunes image including the entire range of graylevels. In both cases, we kept constant the size of the hologram at 512 by 512 pixels. To be consistent, the size of the image to be reconstructed was set to 128 by 128 pixels for the Lee coding, and 256 by 256 pixels for the Island coding. Let's consider 3 cases:

1- Lee coding with 9 levels

2- Island coding with 9 levels

3- Island coding with 201 levels

In Fig. 13a and Fig. 13b are shown the Lee coding 9-levels original image and the simulated reconstructed image. The resulting image is noisy with a low dynamical range for the reconstructed grayscales due to the only 9-levels quantification of the hologram. The binary CGH as well as a detail are in Fig. 13c,d.

In Fig. 14a and Fig. 14b are shown the Island coding 9-levels original image and the simulated reconstructed image. The resulting image is closer to the original, but the dynamical range for the reconstructed grayscales is still limited. The grayscale CGH as well as a detail are in Fig. 14c,d. Note the average value at the mid-gray level for the Island hologram.

In Fig. 15a and Fig. 15b are shown the Island coding 201-levels original image and the simulated reconstructed image. The resulting image is nearly identical with the original, with a full dynamical range for the reconstructed grayscales, thanks to the high number of quantification levels. The contrast of the image is as well maximized. The grayscale CGH as well as a detail are in Fig. 15c,d. Note the average value at the mid-gray level for the Islandl hologram. 

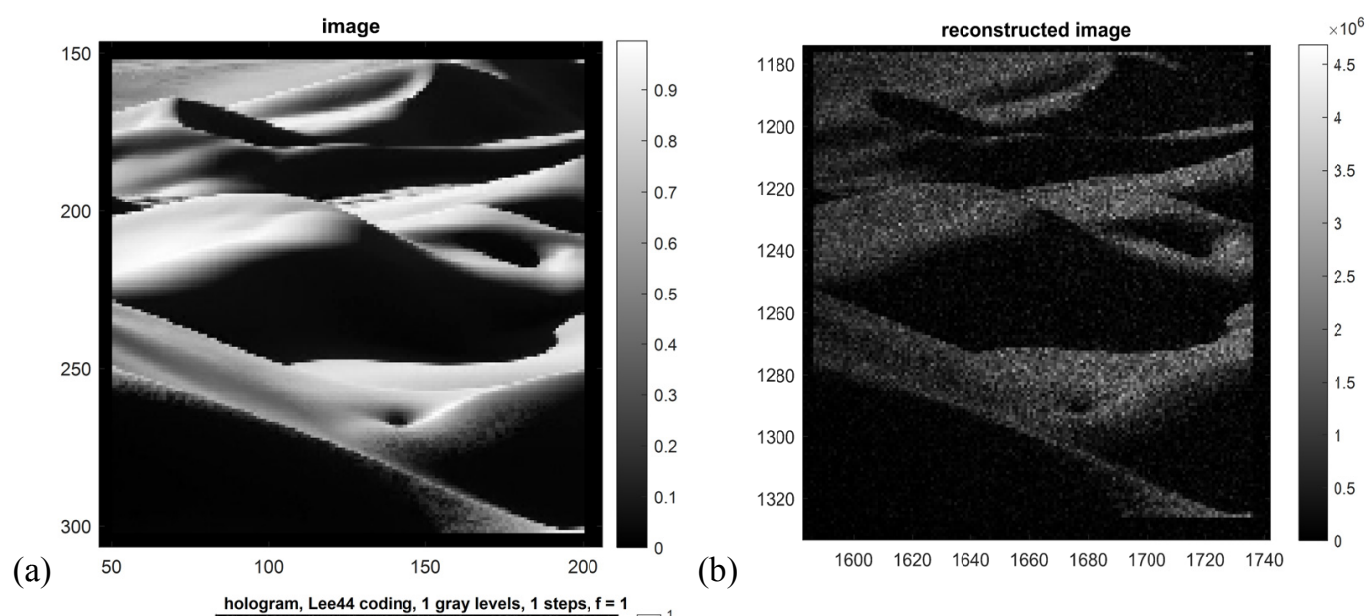

(c)
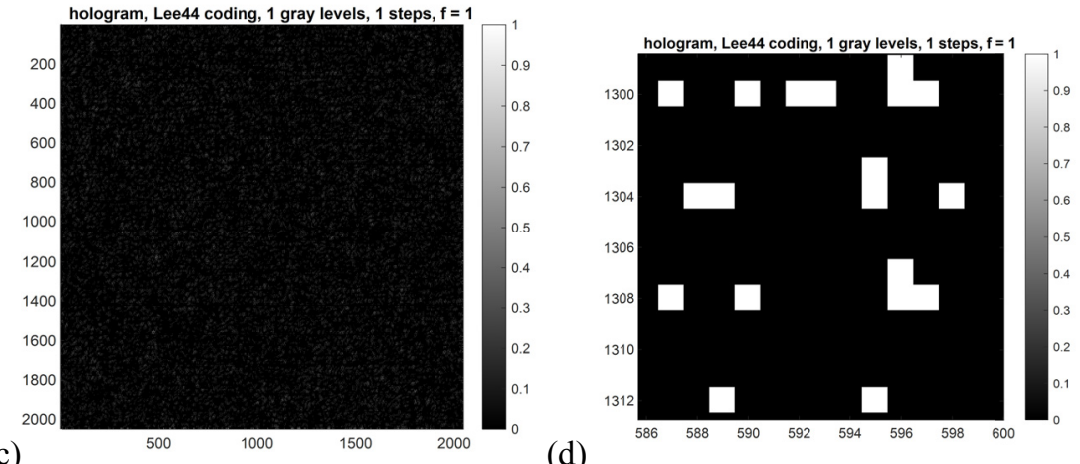

Fig. 13: Lee-coded 9 levels hologram; (a) Original image, (b) reconstructed image, (c) hologram, (d) detail of the hologram.

(a)

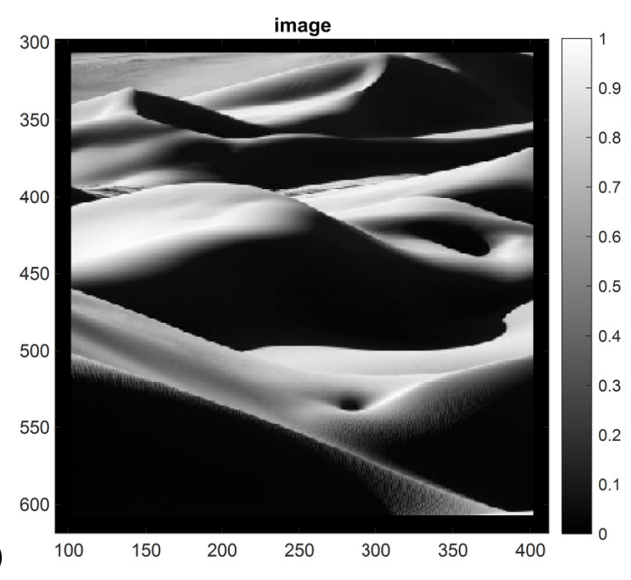

(c)

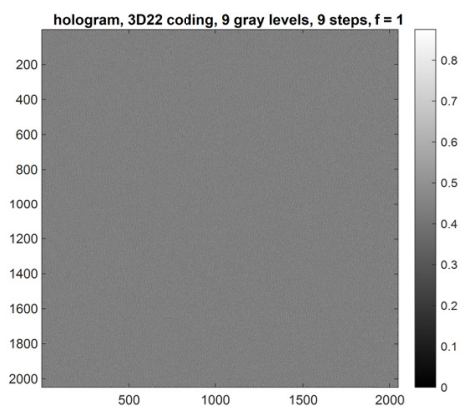

(d)

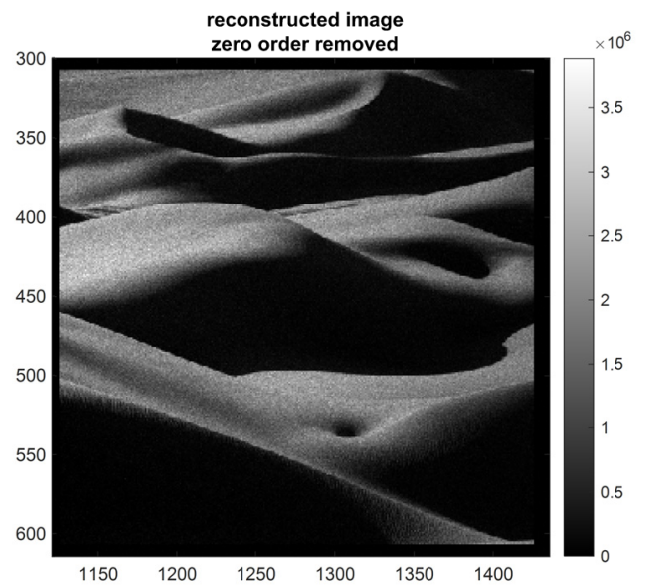

(b)

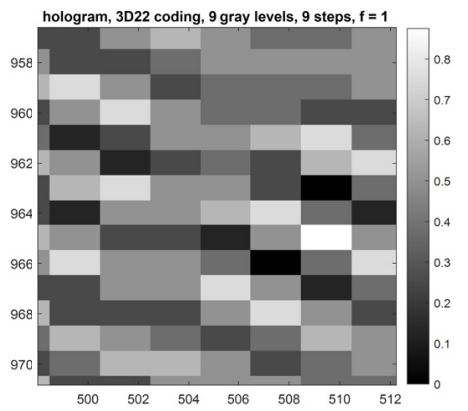

Fig. 14: Island-coded 9 levels hologram; (a) Original image, (b) reconstructed image, (c) hologram, (d) detail of the hologram. 

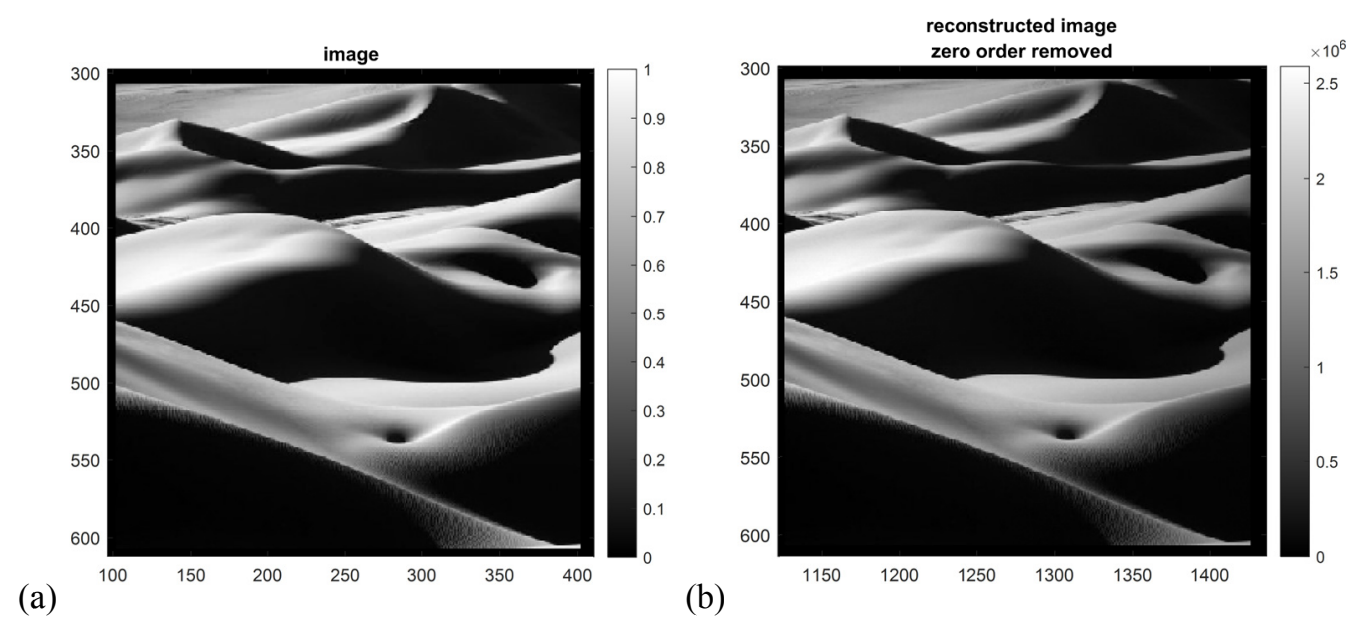

(b)

(c)
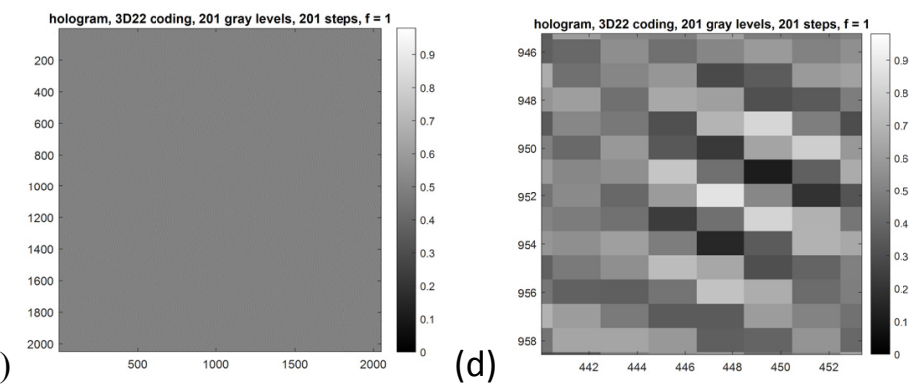

Fig. 15: Island-coded 201 levels hologram; (a) Original image, (b) reconstructed image, (c) hologram, (d) detail of the hologram.

Simulation of grayscale images with our new Island coding scheme shows a much better reconstruction (resolution, fidelity, contrast) thanks to the quantification of the transparency than the Lee algorithm commonly used.

\section{CONCLUSION}

Computer generated holograms are well suited for optical testing and wavefront shaping. Up to now amplitude holograms have only been recorded in binary format, and our method increases their quality by enabling the realization of stepped holograms with discrete gray levels.

We have proposed a new Fourier CGH coding scheme, called Island algorithm, leading to a quantification exceeding 1000 within a smaller cell size of $2 \times 2$ pixels. This code has been implemented for the BATMAN-instrument logo for visiting very different spatial frequencies. The CGH has been recorded with our DMD-based set-up, leading to a 1000x1000 pixels hologram written on a photochromic plate. The reconstruction of the recorded CGH leads to images with a perfect fidelity in shape and intensity, for any single pixel of the original object. Our proposed code exhibits a much higher resolution, a better compacity and an increased throughput, in comparison with the current Fourier CGHs. Grayscale objects have been simulated with our new coding scheme, and show a much better reconstruction (resolution, fidelity, contrast) than the Lee algorithm commonly used.

These results reveal the high potential of this method for generating programmable/rewritable CGHs. The next step will be to code more complex holograms as well as wavefronts containing phase information. We will also use our method to create beam shapers including apodizers, using the same protocol as for stepped CGHs.

\section{ACKNOWLEDGMENTS}

This work has been partly funded by the European Union FP7-OPTICON 2 program. 


\section{REFERENCES}

[1] C. Pruss, S. Reichelt, H. J.Tiziani and W.Osten, ”Computer-generated holograms in interferometric testing, “ Opt. Eng. 43 (11), 2534-2540 (2004)

[2] Brown, B. R. and Lohmann, A. W., “Computer-generated Binary Holograms,” IBM J. Res. Dev. (1969)

[3] Y-T. Lu, C-S. Chu, H-Y. Lin, "Characterization of the gray-scale photolithography with high-resolution gray steps for precise fabrication of diffractive optics, “ Opt. Eng. 43(11) 2666-2670 (2004)

[4] N. Luo, Y. Gao, S. He, Y. Rao, “ Research on exposure model for DMD-based digital gray-tone mask ,” Proc SPIE Vol. 7657, 7657 12-1 (2010)

[5] Romain Alata, Giorgio Pariani, Frederic Zamkotsian, Patrick Lanzoni, Andrea Bianco, Chiara Bertarelli, "Programmable CGH on photochromic plates coded with DMD generated masks", Optics Express, Vol. 25, n 6, (2017), https://doi.org/10.1364/OE.25.006945

[6] Frederic Zamkotsian, Giorgio Pariani, Romain Alata, Luca Oggioni, Patrick Lanzoni, Chiara Bertarelli, Andrea Bianco, "The island CGH, a new coding scheme: concept and demonstration", Optics Express, Vol. 27, Issue 19, pp. 26446-26458, (2019) https://doi.org/10.1364/OE.27.026446

[7] F. Zamkotsian, P. Spano, P. Lanzoni, H. Ramarijaona, M. Moschetti, M. Riva, et al., "BATMAN: a DMD-based Multi-Object Spectrograph on Galileo telescope, " in Proceedings of the SPIE conference on Astronomical Instrumentation 2014, Proc. SPIE 9147, Montréal, Canada, (June 2014)

[8] R.S. Nesbitt, S.L. Smith, R.A. Molnar, S.A. Benton, "Holographic recording using a digital micromirror device," Proc. SPIE 3637, Practical Holography XIII, 12 (March 25, 1999); doi:10.1117/12.343767

[9] Park, M.-C., Lee, B.-R., Son, J.-Y., Chernyshov, O., "Properties of DMDs for holographic displays," J. Mod. Opt. 62(19), 1600$1607(2015)$

[10] C. Bertarelli, A. Bianco, R. Castagna and G. Pariani, "Photochromism into optics: Opportunities to develop light-triggered optical elements, " Journal of Photochemistry and Photobiology C: Photochemistry Reviews 12(2), 106-125 (2011) doi: 10.1016/j.jphotochemrev.2011.05.003

[11] Nan Xie, Yi Chen, Baoli Yao, Ming Lie, "Photochromic diarylethene for reversible holographic recording, " Materials Science and Engineering: B, 138(3), 210-213 (2007)

[12] Yi Chen, Congming Wang, Meigong Fan, Baoli Yao, Neimule Menke, "Photochromic fulgide for holographic recording, “ Optical Materials, 26(1), 75-77 (2004)

[13] Y. Kobayashi and J. Abe, "Real-Time Dynamic Hologram of a 3D Object with Fast Photochromic Molecules, " Adv. Opt. Mater. 4(9), 1354-1357 (2016)

[14] N. Ishii, T. Kato, and J. Abe, "A real-time dynamic holographic material using a fast photochromic molecule" Sci. Rep. 2, 819 (2012)

[15] G. Pariani, C. Bertarelli, G. Dassa, A. Bianco, G. Zerbi, "Photochromic polyurethanes for rewritable CGHs in optical testing, “ Optics Express 5 (19), 4536-4541 (2011)

[16] G. Pariani, A. Bianco, R. Castagna and C. Bertarelli, "Kinetics of photochromic conversion at the solid state: Quantum yield of dithienylethene-based films," Journal of Physical Chemistry A 115(44), 12184-12193 (2011) doi: 10.1021/jp207210p

[17] Romain Alata, Frédéric Zamkotsian, Patrick Lanzoni, Giorgio Pariani, Andrea Bianco, Chiara Bertarelli, "Programmable CGH on photochromic material using DMD generated masks", Proc. SPIE 10546, (2018); https://doi.org/10.1117/12.2292075

[18] F. Zamkotsian, P. Lanzoni, E. Grassi, R. Barette, C. Fabron, K. Tangen, L. Valenziano, L. Marchand, L. Duvet " Successful evaluation for space applications of the 2048x1080 DMD, " in Proceedings of the SPIE conference on MOEMS 2011, Proc. SPIE 7932, San Francisco, USA (2011)

[19] Frederic Zamkotsian, Patrick Lanzoni, Nicolas Tchoubaklian, Harald Ramarijaona, Manuele Moschetti, Marco Riva, Marc Jaquet, Paolo Spano, William Bon, Mathieu Vachey, Luciano Nicastro, Emilio Molinari, Rosario Cosentino, Adriano Ghedina, Manuel Gonzalez, Walter Boschin, Paolo Di Marcantonio, Igor Coretti, Roberto Cirami, Filippo Zerbi, Luca Valenziano, "BATMAN@ @NG: Instrument integration and performance", in Proceedings of the SPIE conference on Astronomical Instrumentation 2018, Proc. SPIE 10702, Austin, USA, (2018), https://doi.org/10.1117/12.2315222

[20] G. Pariani, R. Castagna, G. Dassa, S. Hermes, C. Vailati, A. Bianco and C. Bertarelli, ”Diarylethene-based photochromic polyurethanes for multistate optical memories, “ Journal of Materials Chemistry 21(35), 13223-13231 (2011) doi: $10.1039 / \mathrm{c} 1 \mathrm{jm} 11870 \mathrm{f}$

[21] A. W. Lohmann and S. Sinizinger, "Graphic codes for computer holography," Appl. Opt. 34, 17 (1995)

[22] C. B. Burckhardt, "A simplification of Lee's method of generating holograms by computers," Appl. Opt. 9, 1949-1959 (1970)

[23] C. K. Hsueh and A. A. Sawchuk, "Computer-generated double-phase holograms,” App. Pot. 17, 3874-3883 (1978)

[24] G. Tricoles, "Computer generated holograms: an historical review," Appl. Opt. 26, 4351-60 (1987) 